\section{A Paper}

$$
\text { o.s }
$$

\section{THE DIAGNOSIS AND TREATMENT OF CARCINOMA OF THE COLON* \\ BY}

SIR CHARLES GORDON-WATSON, K.B.E., F.R.C.S. SURGEON TO ST. BARTHOLOMEW'S HOSPITAL; CONSULTING SURGEON TO ST. MARK'S HOSPITAL FOR DISEASES OF THR RECTUM

In the stomach and large intestine we meet only too frequently with the same type of malignant disease, whereas in the small intestine carcinoma is rare. The explanation may be that the stomach and large gut are constantly dealing with solid matter which moves on slowly, and the small gut mainly with fluids quickly passing on. The surface epithelium of the stomach and colon must undergo more active reproduction and be more subject to irritation and mechanical injury than that of the small intestine.

Superficial tumours of the external and internal body covering (that is, skin and mucosa) are derived from alterations of the surface epithelium. We know that certain chemical substances are the exciting cause of certain skin cancers, and we may, perhaps, assume that in the stomach and colon repeated irritation by the contents of the alimentary tract at some weak spot (for example, a gastric ulcer) excites a higher activity in reproduction of the epithelium, a disturbance or irregularity in its reproduction and diminished differentiation, so that the cells grow abnormally and, given some stimulus, as yet unknown, grow independently and form tumours.

If we can accept the theory that mutation in the chromosomes of somatic cells is the causal factor in independent cell growth-that is, malignancy-we must, I think, on the analogy of germ-cell mutations, regard heredity as playing a big part in the independent growth of those particular cells which have been started on their downward path as the result of chronic irritation, either by a specific or a non-specific irritant. The hereditary factor in carcinoma of the colon and rectum is very strong, and it is common to find cancer of the colon and rectum affecting several members of the same family.

The alimentary canal is developed from the fore, mid, and hind guts. The fore gut receives and prepares the food for digestion and absorption by the mid gut, which extends from the jejunum to about the middle of the transverse colon, and in doing so is engaged in active mechanical work on solid material causing wear and tear of its epithelium and consequently active reproduction. The small intestine portion of the mid gut takes up and absorbs the solids, and the large intestine portion absorbs the fluids. Hunger is appeased by the small bowel and thirst by the large. It is essential for the contents of the small bowel to be fluid to suspend the solid particles which are to be absorbed by the valvulae conniventes. By the time the fluid reaches the caecum the only solid material remaining is waste. The hind gut, which extends from the middle of the transverse colon to the rectum, stores and then excretes the waste products of digestion. This portion, like the stomach, is actively engaged with solid material, and its epithelium must be subject to considerable wear and tear.

\section{Early Diagnosis}

Early diagnosis is often difficult. Usually the first symptom noted by the patient is some disturbance in the regularity of the action of the bowels, a certain capriciousness in their action which has replaced a former cast-iron

* Read before the Guildford Division of the British Medical Association, April, 1932. regularity. Associated with this, sometimes, there is a reflex pyloric spasm causing gastric distension, which often misleads both patient and doctor.

When a patient who has passed the meridian of life complains that he no longer enjoys that clockwork regularity with his bowels that has been his privilege and joy through life, it behoves you as his medical man to take something more than passing notice. It may well be that some indiscretion in diet or a disturbance in the normal routine of life has caused a temporary irregularity, which is soon corrected. On the other hand, careful inquiries may elicit no obvious explanation for this rather sudden capricious behaviour of his bowels. In some instances the explanation is that the normal peristalsis of the colon has been disturbed by an outgrowth into the lumen of the bowel. Unfortunately it sometimes happens that the danger signal is not fully appreciated, and the bowels may, after a period of irregularity, adjust themselves to altered circumstances and again become reasonably regular for a time, or sufficiently so to allay suspicions. Then, perhaps, a little later irregularity returns and becomes more persistent, or a sudden attack of subacute obstruction occurs.

A history such as this demands a careful abdominal examination. Many growths of the caecum, transverse colon, and iliac and pelvic colons are palpable, more especially if there is faecal accumulation behind them. A lump in the pelvic colon undetected on abdominal examination is often discovered on bimanual examination or by the sigmoidoscope.

Growths in Proximal Colon.-If a growth arises in the caecum or ascending colon, or the proximal transverse colon, the bowel wall is irritated and peristalsis is increased. The fluid contents of this portion of the gut are pushed on hurriedly into the distal colon, so that diarrhoea results. Later, when narrowing of the bowel commences, peristalsis becomes exaggerated and diarrhoea increases. In these circumstances, sooner or later, some bleeding due to congestion usually occurs, which can be detected in the stools, either by the naked eye or by tests for occult blood. Sometimes the amount of blood lost daily over a long period gives rise to obvious and perhaps severe anaemia, and this loss may be unrecognized because it is small in amount at any one time, and does not attract attention in dark-coloured faeces. An excess of mucus when present, described by the patient as slime, is more likely to be noticed.

Pain in the early stages is rarely complained of, but a sense of discomfort, of erratic and uneasy movements of the bowel-sometimes with gripings, and often with borborygmi-develops as the growth encroaches on the lumen. Frequently the patient is able to localize the seat of trouble from a sensation of wind being held up at a certain spot, followed by the gurgling of borborygmi, which usually signals relief. In these cases visible peristalsis can sometimes be excited by gentle massage of the abdomen, though this is usually a late sign.

Growths in Distal Colon.-A growth in the hind gut or distal half of the colon, unlike the proximal, does not usually excite diarrhoea until ulceration has occurred, but rather tends to delay the passage of faeces and to cause constipation. As the growth advances and begins to constrict the lumen so it tends to produce obstruction more readily than in the proximal colon, where the contents are fluid.

Tumours of the colon often grow to a considerable size without giving rise to noticeable symptoms, and so escape detection. Fortunately growths in the caecum and transverse and pelvic colons are often palpable, and when a growth is causing faecal delay accumulation of faeces above the growth gives rise to a palpable tumour, especially in the pelvic colon. For the most part colon 
growths are low in grade of malignancy, which applies especially to the hypertrophic types, and, unlike growths of the stomach, are slow in giving rise to metastases. To this the colloid growths are an exception. Fortunately, too, the lymphatic and blood supplies correspond, a fact which enables the glands and lymphatics to be removed with the wedge of mesentery corresponding to the resected area.

Speaking generally, carcinomas of the colon, unlike carcinomas of the rectum, rarely attack the young. In a series of seventy-five cases I have met with only two in persons under 30 .

More than half the growths involve the pelvic colon, and in a considerable number of these the diagnosis can be made with the sigmoidoscope. Many growths of the lower end of the pelvic colon are treated as rectal growths by radical excision, or by colostomy alone, and appear in statistics as rectal carcinomas.

While a probable diagnosis can frequently be made on clinical symptoms and sometimes confirmed by palpation, an absolute diagnosis is more often obtained by $x$ rays. $X$ rays may be misleading, and certain fallacies must be borne in mind: (a) faeces or gas in the bowel, or air in the enema may give an apparent filling defect; $(b)$ delay at the recto-sigmoidal junction or at the junction of the pelvic and descending colons may be non-pathological; (c) spasticity may occasionally simulate the filling defect due to a tumour, especially in the distal half: in such instances a further examination is made after injecting atropine to relieve spasm.

The barium meal and the barium enema both have their values. A meal requires repeated observation to visualize a filling defect, whereas an enema visualizes the contour of the colon throughout, or up to the point of obstruction, at one inspection in a much shorter time. The enema, by expanding the colon beyond the normal, emphasizes any irregularity in contour. A limited stenosis, which permits a meal to pass without check, may show a complete block when an enema, given rapidly in the reverse direction, excites spasm. Apart from a filling defect, dilatation above this point is often noted with an enema, especially in the caecum when the growth is in the proximal half. Growths in the pelvic colon, hepatic or splenic flexures, may not be visible in an anterior view owing to overlap, and only recognized in an oblique picture.

An ordinary radiogram of the abdomen, without the use of barium, may be quite useful in showing distension of some portion of the colon with gas. This is sometimes of great value in assisting diagnosis in cases of acute obstruction, if the patient is not too ill to be examined. In such circumstances an obstruction-for example, in the pelvic colon-may come out quite clearly. The colon above the obstruction can often be demonstrated as obviously distended; if the rectum is then inflated with air a shaded interval, representing the obstructing growth, may be obvious between the two distended areas. A radiographic examination of this nature can be carried out quickly, and may serve to differentiate between small and large gut obstruction.

\section{Pathology in Relation to Treatment}

The behaviour of a growth in the colon depends not only on the histological character, but also on the soil. There is no doubt that the family history in these cases is important. Time will not permit me to discuss the relation between adenomata and adenocarcinomata in the colon, but there is good evidence to show that adenocarcinoma frequently develops in an adenoma. We know, too, that multiple adenomatosis of the colon runs in families, and that sooner or later the victims of this disease will develop one or more carcinomata and that these families tend to die out. ${ }^{1}$

It is a noticeable feature of colon growths that the large proliferative type tends to be less malignant and less prone to invade the glands than the small scirrhous type, which ulcerates early; and is inclined to invade the glands in the mesentery, which, like the primary growths, are hard and small when involved. Consequently, large growths with adhesions and without metastases should not be regarded unfavourably, but looked upon as evidence of good resistance. Prognosis must be considered good in this type of case if a satisfactory radical operation can be performed. In some instances large soft glands are encountered in the proliferating or encephaloid type of growth, and it is important to note that these often prove to be inflammatory.

When, however, an adenocarcinoma shows evidence of colloid degeneration the prospects of cure are greatly diminished. In colloid cases growth is more rapid, the peritoneum is invaded early and free fluid quickly forms. Metastasis spreads widely in the glands, which grow to a large size and develop colloid change. If a growth is recognized as colloid in type less risk to life should be taken, when considering the advisability of radical treatment, because prognosis is not good, even with ideal surgical procedures.

\section{TREATMENT}

It is probable that there are few situations in the body where radical operation for carcinoma offers such promising results, especially in early cases. A wide resection and anastomosis is as a rule not difficult. The lymphatics run with the vessels and converge on the apex of the resected mesentery. Lymphatic spread is usually slow, and if colloid cases are excepted the grade of malignancy of adenocarcinoma of the colon is low. This is especially true of the hypertrophic type.

While it is true that in cases favourable for radical surgery the prognosis is good, meticulous care is required for radical surgery to be successful. The blood supply of the colon is not so good as that of the stomach; the contents of the colon are dangerously infective to the peritoneum, and gas distension after resection may be disastrous if there is no safety valve. Risks are far greater than in cases of gastric resection, and statistics from all sources show that the operative mortality rate for resections is high, whereas the ratio of recurrence is low in comparison with gastric resections. In radical surgery for cancer of the colon and rectum a high " operability" rate gives a high mortality rate, and vice versa. If radical surgery is reserved for the freely mobile case, both the operative mortality rate and the ratio of recurrence will be low, and in estimating the value of statistics in these cases we ought to know the percentage treated by radical measures to those treated by palliative measures. This is true for surgery of the rectum as well as for the colon. There can be no doubt that many cases of carcinoma of the colon which are adherent to the parietes, or to other viscera, can be mobilized and resected with good endresults in a percentage of cases, a percentage which is necessarily lower than in freely mobile cases.

\section{Preliminary Drainage in Obstructed Cases}

It must be laid down as an axiom that preliminary drainage reduces the mortality of radical surgery to about half, and that in the absence of preliminary drainage measures must be adopted at the time of resection to prevent post-operative distension and to avoid leakage at the anastomotic line, if distension occurs, by means of a safety valve which will allow the escape of gas and fluid.

It is universally agreed that resection and anastomosis should never be performed in the presence of acute or 
subacute obstruction, and that a preliminary caecostomy is usually the wise course to adopt. Opinions differ as to the respective merits of a so-called " blind " caecostomy (that is, a caecostomy performed without exploration of the abdominal cavity and often under local anaesthesia), and an exploratory laparotomy to locate the seat of obstruction followed by a caecostomy or in some cases colostomy. The main argument against a "blind" caecostomy is the possibility of a mistake in diagnosis. The strongest argument in its favour is that the mortality of the operation, in acute cases, is considerably lower than it is for the exploratory method.

When obstruction is subacute, the case for "blind" caecostomy is not so strong. In some of these a freely mobile growth can be safely marsupialized and the colon drained by the method of Paul, or a variant of this, though it is doubtful if the end-results of cases treated by this type of operation can be compared with planned resections in the quiescent period. It is seldom possible to remove the mesentery as freely as one would desire in a Paul's operation, and the resulting anastomosis will not have that freedom in the abdominal cavity which is desirable and can often be secured by resection in the quiescent period.

\section{Preliminary Caecostomy in Obstructed Cases}

This relieves obstruction, whether acute, subacute, or threatened, and allows free drainage of faecal contents. It prevents recurrent distension with gas and so relieves pain, and allows the muscular coats of the bowel to recover tone after distension. It also relieves secondary infection following obstruction, thus preventing ulceration behind a growth and the risk of perforation, and permits irrigation of the colon behind the growth. By thus relieving obstruction, pain, infection, etc., the general condition of the patient is enormously improved, so that the chances of recovery from a severe resection operation later are much more favourable.

In many instances of carcinoma of the colon such warnings as a medical man might recognize the patient may often ignore, and a sudden acute obstruction may be the first symptom complained of. When this happens it is often a blessing in disguise because it compels preliminary drainage. The gradual onset of obstruction is less favourable, because the bowel does not recover its normal tone and healthy content so readily when distension and the resultant toxaemia has become chronic.

\section{Radical Treatment in the Quiescent Period}

In the absence of any active or threatened obstruction the question of preliminary drainage, either by caecostomy or short circuit before resection, has always to be considered. Each case must be judged on its merits. If the patient, by virtue of age or infirmity, is not regarded as a good operative risk, "safety first" should be the guiding rule. In cases of doubt, when the growth is in the caecum, ascending colon, or hepatic flexure, the risks of resection will be diminished by a preliminary ileo-colostomy (transverse colon). If the growth is in the splenic region or descending colon a preliminary short circuit between transverse and sigmoid should be performed if a two-stage operation is indicated.

When the growth is in the transverse, iliac, or pelvic colon I prefer a one-stage operation with proximal drainage at the time of resection, and in resections of the pelvic colon aided by a long rectal tube passed through a stretched sphincter. These short-circuit operations, though occupying less time than the combined resection-anastomosis, are not without risk, and sometimes, owing to adhesions which may follow, hamper the surgeon in his subsequent resection. It must not be forgotten that two operations mean two anaesthetics and a longer recumbent period, with their attendant risks and disadvantages in elderly people. It is difficult sometimes, when carrying out a two-stage resection, to avoid leaving blind ends to the anastomosis, which may act as stagnant reservoirs causing pain and discomfort. I have twice operated for this condition and removed the superfluous ends.

On the whole, anastomosis followed by resection has more disadvantages than anastomosis and resection in one stage, and should not be employed in cases regarded as good operative risks. It seems probable that in the bad risks (we are not considering here cases with obstruction), preliminary drainage, followed by a one-stage resection-anastomosis, should be regarded as the operation of choice, and there are those who feel that preliminary drainage should be the standard practice for all cases, whether good or bad risks, and none can quarrel with their view, which is justified by statistics of operative mortality.

I have, however, had so much success, in the absence of any degree of obstruction, with the one-stage resectionanastomosis and proximal drainage that I do not think I could get better results with preliminary drainage, which, however, I never hesitate to employ when obstruction in any form exists, or has previously been noted.

I ought to refer to those cases involving the lower portion of the pelvic colon which are too low to permit of resection and anastomosis. Some surgeons treat these by abdomino-perineal resection. I believe, however, that there is no justification for removing the rectum in these cases, provided that it is possible to clamp and divide the pelvic colon below, and some two inches (more if possible) clear of the growth. In these circumstances the lower end is closed and the pelvic colon removed, leaving a permanent colostomy. This operation becomes necessary sometimes with a short fatty pelvic colon, when the growth is high enough in the pelvic colon to permit of resection, and yet when removed does not leave enough colon to allow an anastomosis without tension. When there is much fat in the mesentery and in the appendices epiploicae an anastomosis is more difficult. It is not so easy to secure accurate apposition, and there is more risk of defective blood supply. A live patient with a colostomy is better than one dead with a faulty anastomosis. Generally speaking, a lateral anastomosis is safer than end-to-end, because suturing is easier and wider areas of peritoneum can be opposed to one another. At the same time, the opening can be made wide enough to allow for any subsequent contraction and wider than the normal diameter of the resected portion, so that subsequent gaseous distension does not unduly stress the sutured area.

In resections of the pelvic colon an end-to-end anastomosis is usually adopted, as it is considered wise to remove as much colon above and below as is possible under the circumstances-that is, consistent with an end-to-end anastomosis. When this can be done while the cut ends are outside the peritoneal cavity, the operation is easier and safer than when conducted within the abdomen. Subsequent safety from leakage is helped considerably by the use of a large rectal tube passed through the sphincter (which has been previously stretched) and into the anastomotic area.

\section{Palliative Operations}

In the absence of visceral metastasis, and visceral metastasis is usually a late manifestation, the majority of cases of cancer of the colon can be treated on radical lines if there is no obstruction or provided that obstruction has been relieved, and provided also that the patient is a fit subject for the treatment proposed. A colon growth which at first sight may appear hopelessly fixed can usually be mobilized with care to allow free resection, though it may be necessary to remove a portion of another 
viscus with it, and the operation may be difficult and long.

As already stated, the higher the percentage of cases treated radically, as against palliative treatment, the higher is the operative mortality rate, because it is inevitable that many of the more complicated cases must fail to survive heroic procedures. Nevertheless, the successful cases fully justify heroic attempts, and many cases that at first look hopeless are permanently cured by bold surgery. The position with regard to gastric carcinoma is quite different.

When visceral metastasis, or general dissemination of the peritoneum, has occurred much may be done to give relief by a short circuit when this is possible. It may sometimes be doubted whether a nodule felt in the liver, which cannot be seen, is a metastasis or not. In such instances the patient should be given the benefit of the doubt. It is certainly better to remove a growth which may cause much distress to the patient and leave a nodule in the liver which may ultimately cause the patient's death, than to leave behind a growth which must ultimately kill the patient, having mistaken an angionoma fibroma, etc., for a secondary deposit.

\section{The Technique of Resection}

It is an unfortunate thing that, whereas the ultimate prognosis of radical surgery for malignant disease of the colon is so good in comparison with that of many other regions of the body, the technical difficulties are such that many, who could be cured if surgery was well and truly done, fail to survive the operation. So great are the risks of anastomotic leak, either by infection spreading through the suture line, or through inadequate blood supply, or a combination of both, that some surgeons deliberately avoid a one-stage operation (even with preliminary drainage) and prefer a two-stage method such as Paul devised.

Devine of Melbourne has introduced a modification of Paul's method. An enterotome, shaped to encourage peritoneal adhesions along the line of the anastomotic opening about to be produced, is employed at the time of marsupialization, and the two divided ends, after turning back a cuff of the peritoneal and muscular coats of the bowel, are closed down over the enterotome, except for the necessary drain. In this way the final closure is accelerated and much time saved. In this type of operation, under favourable conditions as regards the amount of bowel available to be drawn outside, I have found that a direct lateral anastomosis can sometimes be made at the first operation, while the two cut ends are left open and fixed to the parietes after the growth has been resected. The use of the enterotome is thus avoided, but the operation takes longer and is not suitable for obstructed cases.

The risks referred to above can only be countered by the most careful attention to detail. Free exposure of the area to be dealt with is essential, and, except when operating on the transverse colon, can best be secured by a long lateral oblique incision. In the case of growths at the splenic angle which are often adherent to spleen, stomach, etc., the best incision is along the distal half of the twelfth rib, and extended forwards. The distal half of the rib is resected, and an admirable exposure then secured.

The next important point is to protect the surrounding viscera from possible contamination. To this end Pringle and others devised and adopted the so-called aseptic method of end-to-end anastomosis, which, though it has definite advantages as regards contamination, is not proof against risks of haemorrhage from the cut ends. In one instance where $I$ adopted this method in a non-malignant case a serious haemorrhage into the bowel occurred.
De Martel has devised a special clamp which diminishes the risks of contamination during resection, though the clamp is not very suitable for end-to-end anastomosis.

I have a strong preference for the lateral anastomosis when possible. To open the bowel I use the electric cautery, which prevents haemorrhage at the time and assists the sutures in preventing reactionary haemorrhage later on. The line of anastomosis is always protected by suturing appendices epiploicae or omental tags accurately round the junction. A small safety valve (using a No. 12 catheter) is made with the cautery proximal to, and clear of, the anastomosis, and this is brought up to the wound after omentum has been sutured round it. In pelvic colon resections a large rectal tube is substituted for the safety valve, though sometimes both are employed. As far as it is possible to do so, such areas of the parietes as have been bared of peritoneum should be obliterated or covered by omentum, and every bleeding point carefully secured. Any collection of blood following operation adds to the risks of infection or adhesions later, and any bare area to some adhesions which may give rise to complications later, sometimes wrecking an otherwise successful operation.

I need hardly add that adequate drainage to the site of anastomosis is essential. It is also advisable to drain the abdominal wall; the tissues of the abdominal wall are easily infected, and secondary abscess is not uncommon.

\section{Statistics}

I have taken ten years' statistics-two periods of five years. The year 1926 has been left out, as only one case was operated on in that year. All cases of pelvic colon growths have been included which were, or might have been, if operable, treated by abdominal resection. Cases with growths in the region of the recto-sigmoidal junction treated by excision of the rectum or by colostomy, though anatomically growths of the colon, have been excluded. Those cases, recorded as obstructed, are cases operated on for acute obstruction. Several of these were desperate cases, some with perforation of the caecum. In three of them resections were performed at a later date after obstruction had been relieved. In the remainder, peritoneal or visceral metastasis prevented radical procedures. Cases with subacute obstruction are not included under this heading.

It is of some interest to compare the two five-year periods. It may be chance only that the percentage of acute cases dealt with is lower in the second period, or it may be an indication of earlier diagnosis. The improvement in the statistics for resected cases is noticeable. This no doubt is partly due to increased experience and improvement in technique. It may be noted that whereas in the first period there were two deaths attributed to shock, there were none in the second. The more frequent use of blood transfusion before and after operation, combined with gas-oxygen-ether anaesthesia (and in some the use of avertin, which reduces the ether to a minimum), has considerably reduced the risks of shock in severe operations.

In these statistics all causes of death after operation, and before the patient leaves the hospital, are included under " operation mortality." One of the resected cases, a man over 70, made an excellent surgical recovery and died rather suddenly from heart failure a week later. Another patient who appeared to be going to recover died of pulmonary embolism on the eighth day. Of the three deaths from peritonitis, two were due either to defective blood supply or imperfect suturing of the anastomosis, and the other to contamination, as the anastomosis was intact. In the case of death from ileus, dilated stomach, and peritonitis, the anastomosis was sound; 
it was found at operation that the patient's colon had not been properly cleared out, and there was a mass of faeces proximal to a growth in the splenic flexure. It would have been much wiser to delay resection in this case until after drainage and irrigation of the colon.

It is noticeable that there was only one death in the cases of palliative short circuit, this being due to bronchopneumonia. This favourable mortality rate in comparison with that of resection and anastomosis suggests that interference with efficient blood supply of the line of anastomosis by resection of the mesentery is the main factor in anastomotic leak and a fatal issue, and one that must be guarded against most carefully. The risks of imperfect blood supply in end-to-end anastomosis are certainly greater than in lateral. I generally employ the lateral method for this reason, except when it is not possible, as is most often the case with the pelvic colon. It is certain that the mortality for resections in the distal half of the colon, and especially the pelvic colon, have a higher mortality than those of the proximal colon.

I have not attempted in this paper to deal with endresults. The cases in the first series referred to have not yet been followed up. The real test of good statistics is end-results - that is, the percentage of five-year cures of all cases operated on, exclusive of those found to have visceral metastasis at operation. It is not enough to show a good percentage of resection recoveries; these cases should also form a high percentage of the total operation. For example, one surgeon might explore ten cases of carcinoma of the colon, all free from metastasis, and resect three of them (all early cases) with no death, and these three might survive five years, while the remainder with short circuit or colostomy survive only a few months or years. Another surgeon meeting the same series of cases might be bold enough to resect seven of the ten, with one death, and four of the surviving six might live five years. The statistics of the former surgeon would be: operation mortality, nil, percentage of five-year cures, 30 ; and the statistics of the latter: operation mortality, 14 per cent., percentage of five-year cures, 40. When it is recognized what can be accomplished in colon surgery the surgeon who errs on the side of boldness will save more cases in the long run.

\begin{tabular}{|c|c|c|c|c|c|c|c|c|}
\hline \multicolumn{3}{|c|}{$1921-1925$} & \multicolumn{3}{|c|}{$1927-1931$} & \multicolumn{3}{|c|}{ Total } \\
\hline 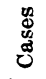 & 离 & $\begin{array}{c}\text { Percentase } \\
\text { Mortality }\end{array}$ & 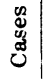 & $\begin{array}{l}\text { Operation } \\
\text { Mortality }\end{array}$ & $\begin{array}{l}\text { Percentage } \\
\text { Mortality }\end{array}$ & $\left|\begin{array}{l|}0 \\
0 \\
0 \\
0 \\
0 \\
0\end{array}\right|$ & $\begin{array}{l}\text { Operation } \\
\text { Mortality }\end{array}$ & \begin{tabular}{|l} 
Percentage \\
Mortality
\end{tabular} \\
\hline 41 & 9 & 21.9 & 24 & 7 & 20.5 & 75 & 16 & 21.0 \\
\hline \multicolumn{9}{|c|}{ Obstruced } \\
\hline $11^{*}$ & $4 b$ & 36.3 & 5 & $3^{c}$ & 60.0 & 16 & 7 & 43.7 \\
\hline \multicolumn{9}{|c|}{ Resected } \\
\hline 22 & $5^{d}$ & 23.7 & 23 & $3^{e}$ & 13 & 46 & 8 & 17.7 \\
\hline \multicolumn{9}{|c|}{ Anastomosis only } \\
\hline 6 & 10 & nil & 3 & 1 & 33.3 & 9 & 1 & 11.0 \\
\hline \multicolumn{9}{|c|}{$\begin{array}{c}\text { Colostomy only } \\
\text { (non-obstructed) }\end{array}$} \\
\hline 2 & 0 & nil & 3 & 0. & nil & 5 & 0 & nil \\
\hline
\end{tabular}

a Thres cases resected later and included in resections; remainder colon drainage or abdominal drainage.

b Two perforated at time of operation.

c One patient died under anaesthetic before operation.

d Two shock; 2 peritonitis ; 1 cardiac failure.

e One peritonitis; 1 peritonitis and ileus; 1 pulmonary embolism.

\section{Conclusion}

I have not attempted in this paper to do more than give expression to my own experience. I am conscious of the fact that few surgeons meet with sufficient cases to get that volume of experience which is really necessary to become expert. Carcinoma of the colon is a far less common disease than carcinoma of the rectum, and I have excised something like five times as many rectums as I have resected colon growths. As the volume of cases dealt with increases so the full range of possible pitfalls and complications becomes unfolded, and the surgeon learns how to avoid these, and to improve both his technique and his statistics. I know that this is true in my case for excision of the rectum, and I feel confident that with continued experience in the treatment of carcinoma of the colon I shall be able to improve both my technique and my results, more especially if, by stimulating interest in early diagnosis, cases come for treatment at an early stage. In this connexion it is worth noting that my operative mortality of resections from 1921 to 1925 was 23 per cent., and for 1926-31 only 13 per cent., and I may add that the last thirteen cases in this series survived operation.

${ }^{1}$ Dukes, C.: The Hereditary Factor in Polyposis Intestini, Cancer Review, 1930, v, 241.

\section{NOTES ON 1,500 CASES OF ASTHMA}

BY

JAMES ADAM, M.A., M.D.

LARYNGOLOGIST TO GLASGOW CORPORATION HOSPITALS AND TO LANARKSHIRE COUNTY HOSPITALS

In this survey only such facts as may throw light on some of the more important points raised in recent controversy will be touched upon. It is particularly essential that in asthma figures should be closely correlated to clinical facts, as neglect of this has led to misleading statistics.

\section{Diagnosis}

Chevalier Jackson ${ }^{1}$ wisely says that all is not asthma that wheezes, and that " asthmatoid" wheeze is a frequent symptom of inspirated foreign body with missed diagnosis; but when later he suggests that asthmatics should be regularly bronchoscoped, one may reply that there are simpler and more acceptable means of settling the diagnosis and, usually, of giving relief. Adrenaline will not ease " asthmatoid" wheeze, but usually gives prompt relief in genuine asthma. Eosinophils crowd the sputum in asthma, are scanty in the " asthmatoid " and mostly replaced by polynuclears; they are usually above 4 per cent. in asthmatic blood, below it, with probably a polynucleosis, in the " asthmatoid." Wheeze plus eosinophilia is asthma, wheeze without it demands scrutiny of the diagnosis; wheeze plus tough viscid sputum crowded with eosinophils is asthma ; wheeze plus purulent sputum with few eosinophils but with many polynuclears is not simple asthma. It is too often forgotten that asthmatic sputum is not purulent. Even the clinical history, as these records abundantly show, will prove the diagnosis. Dyspnoea coming on typically between 2 and 4 a.m. or on waking, or after food, is not asthmatoid but asthmatic. Attention to these points has in this series excluded other dyspnoeic conditions-renal, cardiac, and arteriosclerotic.

\section{EOSINOPHILIA}

Increase of eosinophils in the blood beyond 4 per cent. (on an average 8.4 per cent. in 68.5 per cent. of the cases) has been the rule. Of the remaining 31.5 per cent., threefourths had no asthma at the time of count, so that correlating count with wheeze we find eosinophilia in 80 per cent. This is much nearer the truth than Gram's misleading figure of $\mathbf{4 4}$ per cent., quoted recently in this Journal. ${ }^{2}$ There is no better guide to diagnosis than the eosinophil count, and no better indication of progress under treatment than its fall-a patient free from wheeze but with a high count is a candidate for a fresh attack. The percentage may vary quickly. It mostly rises before and falls after the attack; in menstrual asthma it rises before and falls after the flow. It rises within an hour after ingestion of 